\title{
Hidden Local Symmetry and Chiral Effective Theory for Vector and Axial-vector Mesons
}

\author{
Yong-Liang $\mathrm{Ma}^{\dagger}$, Qing Wang* and Yue-Liang $\mathrm{Wu}^{\dagger}$ \\ ${ }^{\dagger}$ Institute of Theoretical Physics, Chinese Academy of Sciences, Beijing 100080, China \\ *Department of Physics, Tsinghua University, Beijing 100084, China
}

(Dated: November 18, 2018)

\begin{abstract}
In this paper, we present the full Lagrangian of mesons (pseudoscalars, vectors and axial-vectors) to $O\left(p^{4}\right)$ by using the explicit global chiral symmetry and hidden local symmetry in the chiral limit. In this approach, we see that there are many other terms besides the usual eleven terms given in the literature from hidden local symmetry approach. Of particular, there are some terms in our full results which are important for understanding the vector meson dominance and $\pi-\pi$ scattering and providing consistent predictions on the decay rates of $a_{1} \rightarrow \gamma \pi$ and $a_{1} \rightarrow \rho \pi$ as well as for constructing a consistent effective chiral Lagrangian with chiral perturbation theory. It is likely that the structures of the effective chiral Lagrangian for $O\left(p^{4}\right)$ given in the literature by using hidden local symmetry are incomplete and consequently the resulting couplings are not reliable. It is examined that the more general effective chiral Lagrangian given in present paper can provide more consistent predictions for the low energy phenomenology of $\rho-a_{1}$ system and result in more consistent descriptions on the low energy behavior of light flavor mesons.
\end{abstract}




\section{INTRODUCTION}

The strong interaction is believed to be described by $S U(3)$ gauge theory. As an asymptotic free theory, it has successful applications in high energy region (i.e., $E>1 G e V$ ), but in low energy region (i.e., $E<1 G e V$ ), one cannot make ordinaty perturbation calculations since, in this region, the coupling constant becomes large. To describe the physics of strong interaction in low energy region, one may develop some effective theories which reflect the symmetries and symmetry breaking in this energy region. In this note, we focus on the chiral effective Lagrangian theory.

The basic idea of chiral effective Lagrangian theory can be described as follows: Compared with the scale where the nonperturbative effects become important, the masses of the lightest three flavor quarks $(u, d$ and $s)$ are smaller than the QCD scale $\Lambda_{Q C D}$. When neglecting the masses of these quarks, QCD Lagrangian possesses an $U(3)_{L} \times U(3)_{R}$ flavor chiral symmetry. The chiral effective theory was first proposed by S.Weinberg in 1979 [1], where the effective theory of two light flavor quarks $(u$ and $d$ ) was built. Later on, the effective theory of two $(u$ and $d)$ and three $\left(u, d\right.$ and $s$ ) flavor cases was studied systematically up to $O\left(p^{4}\right)$ in $[2]$. Besides the normal parity section, there are anomalous sections in the effective theory [3] [4].

In addition to the pseudoscalar mesons, there are also vector and axial-vector mesons in the meson spectrum. How to build an effective theory of vector and axial-vector mesons was discussed by many authors. In the literatures, many methods were used, such as matter field method [5], massive Yang-Mills method [6] [7], anti-symmetric tensor field method [2] 8], hidden local symmetry method 99 [10] [11] and QCD Green function approach 12]. In general, one should also consider the light scalar mesons which have been shown[13] to play an important role for understanding the dynamically spontaneous symmetry breaking of the chiral symmetry $U(3)_{L} \times U(3)_{R}$. Of particular, a chiral effective Lagrangian with scalars can be derived from integrating out the quark and gluon fields by using a new symmetrypreserving loop regularization method[14], and the gap equations have been found to be resulted from minimal conditions of effective potential for the scalar fields. It then predicts the existence of $\sigma$ and $\kappa$ scalars as the nonet scalar mesons which can be regarded as composite Higgs bosons with a consistent mass spectra to the current experimental data [13]. For simplicity, we will not include the scalar mesons in this paper.

The hidden local symmetry method is based on a popular idea that the nonlinear $\sigma$ model 
based on the manifold $G / H$ is gauge equivalent to the $\sigma$ model based on $G \times H_{\text {local }}$ and the gauge bosons correspond to the local symmetry can be regarded as composite bosons. In our present consideration, we will use the extended hidden local symmetry where $G=U(3)_{L} \times$ $U(3)_{R}$ for three light flavor $(u, d$ and $s)$ case. This model is gauge equivalent to the nonlinear $\sigma$ model based on the manifold $G / H$ [10] [15]. Of course, there are maybe contributions to the coefficients of the nonlinear $\sigma$ model from the Yang-Mills-type self-interaction of the hidden symmetry [16], but we will not consider this case in this note. In the hidden local symmetry method, the vector and axial-vector mesons are treated as combinations of the dynamical gauge bosons of hidden local symmetry $G_{\text {local }}=\hat{U}(3)_{L} \times \hat{U}(3)_{R}$ as suggested in [10]. But after a careful check, we will see that there are many terms including three important terms to $O\left(p^{4}\right)$ were missed in the literature [10], it is these three important terms that can cancel the strong momentum dependence of the $\rho-\pi-\pi$ coupling $f_{\rho \pi \pi}$ and also it is these three terms that can ensure the $\rho$ - meson dominance in $a_{1} \rightarrow \gamma \pi$ decay and result in consistent predictions on the decay rates $a_{1} \rightarrow \gamma \pi$ and $a_{1} \rightarrow \rho \pi$. Of particular, these new terms play an important role for understanding the $\pi-\pi$ scattering [2] [17] [18], or more generally, the meson-meson scattering.

The paper is organized as follows, in section II, we will give a simple but complete description of hidden local symmetry. In section III, after list the fourteen important terms of the effective chiral Lagrangian, we choose a special gauge, i.e., unitary gauge, and explicitly present a gauged Lagrangian explicitly. In section IV, it is shown that with an appropriate gauge fixing condition for the hidden local symmetry, fourteen parameters appearing in the more general effective Lagrangian based on the explicit global chiral symmetry and hidden local chiral symmetry can be uniquely extracted when comparing it with the effective Lagrangian of chiral perturbation theory. The relevant low energy phenomenologes of $\rho-a_{1}$ system, such as universality of the $\rho$-meson coupling, vector meson dominance, the $\rho-\pi-\pi$ coupling $f_{\rho \pi \pi}$, the KSFR relation $m_{\rho}^{2}=f_{\rho \pi \pi}^{2} f_{\pi}^{2} / 2$, etc., are discussed in section V. Our conclusions and remarks are presented in section VI. The full Lagrangian up to $O\left(p^{4}\right)$ is presented in the Appendix. 


\section{HIDDEN LOCAL SYMMETRY}

In chiral limit, the vector and axial-vector mesons can not be introduced as gauge bosons via gauging the above global chiral symmetry $G_{\text {global }}=U(3)_{L} \times U(3)_{R}$, otherwise there exists, according to the Higgs mechanism, no independent degrees of freedoms for the Goldstonelike pseudoscalar mesons. On the other hand, the chiral gauge boson couplings to the light quarks must be invariant under the transformation of the global chiral symmetry $G_{\text {global }}$ as the original QCD theory does. It is then motivated to introduce hidden local chiral symmetry $G_{\text {local }}=\hat{U}(3)_{L} \times \hat{U}(3)_{R}$ associated with the chiral gauge bosons $\hat{A}_{L}$ and $\hat{A}_{R}$. After the spontaneous breaking of the global chiral symmetry $G_{\text {global }}$, the Goldstone-like pseudoscalar mesons are generated, the chiral gauge bosons associated with the hidden local gauge symmetry also turn out to be vector and axial-vector mesons via an appropriate choice

of the gauge transformation of the hidden local symmetry $G_{\text {local }}$. Such a gauge choice breaks the hidden local chiral symmetry and generates the masses of the vector and axial-vector mesons. In this paper, we are limited to consider the case of chiral limit and will not discuss the gauge anomalous section.

Let's begin with introducing the necessary fields for constructing the chiral Lagrangian which are covariant under the global chiral symmetry $G_{\text {global }}$. The chiral Lagrangian is expected to describe the Goldstone-like pseudoscalar mesons, vector mesons and axial-vector mesons which arise from the gauge bosons of the local chiral symmetry $G_{\text {local }}$.

Corresponding to the global chiral symmetry $G_{\text {global }}$, we introduce the local chiral symmetry $G_{\text {local }}$. In this case, we have the nonlinear chiral fields $\hat{\xi}_{L}(x) \in U(3)_{L} \times \hat{U}(3)_{L}$ and $\hat{\xi}_{R}(x) \in U(3)_{R} \times \hat{U}(3)_{R}$, which transform as

$$
\begin{array}{ll}
\hat{\xi}_{L}(x) \rightarrow g_{L} \hat{\xi}_{L}(x) G_{L}^{\dagger}(x) ; & g_{L} \in U(3)_{L},
\end{array}
$$

We also have nonlinear chiral field $\xi_{M}(x) \in G_{\text {local }}$, its transformation property is

$$
\xi_{M}(x) \rightarrow G_{L}(x) \xi_{M}(x) G_{R}^{\dagger}(x) ; \quad\left(G_{L}(x), G_{R}(x)\right) \in \hat{U}(3)_{L} \times \hat{U}(3)_{R}
$$

With the above nonlinear chiral fields, we can construct nonlinear field $U(x) \in G_{\text {global }}$ as follows

$$
U(x) \equiv \hat{\xi}_{L}(x) \xi_{M}(x) \hat{\xi}_{R}^{\dagger}(x)
$$


and its transformation property under the full group $G_{\text {global }} \times G_{\text {local }}$ is

$$
U(x) \rightarrow g_{L} U(x) g_{R}^{\dagger} ; \quad\left(g_{L}, g_{R}\right) \in G_{\text {global }}
$$

From the above, we can also see that the transformation properties of gauge fields $\hat{A}_{L}$ and $\hat{A}_{R}$ corresponding to local chiral symmetry $G_{\text {local }}$ are

$$
\begin{aligned}
& \hat{A}_{L} \rightarrow \hat{A}_{L}^{\prime}=G_{L}(x)\left(\hat{A}_{L}+i \partial\right) G_{L}^{\dagger}(x) \\
& \hat{A}_{R} \rightarrow \hat{A}_{R}^{\prime}=G_{R}(x)\left(\hat{A}_{R}+i \partial\right) G_{R}^{\dagger}(x)
\end{aligned}
$$

Similarly, we can construct chiral gauge bosons

$$
\begin{aligned}
& a_{L}(x)=\hat{\xi}_{L}(x)\left(\hat{A}_{L}(x)+i \partial\right) \hat{\xi}_{L}^{\dagger}(x) \equiv \hat{\xi}_{L}(x) i D \hat{\xi}_{L}^{\dagger}(x) \\
& a_{R}(x)=\hat{\xi}_{R}(x)\left(\hat{A}_{R}(x)+i \partial\right) \hat{\xi}_{R}^{\dagger}(x) \equiv \hat{\xi}_{R}(x) i D \hat{\xi}_{R}^{\dagger}(x)
\end{aligned}
$$

their transformation properties under the full chiral symmetry $G_{\text {global }} \times G_{\text {local }}$ are

$$
a_{L}(x) \rightarrow g_{L} a_{L}(x) g_{L}^{\dagger}, \quad a_{R}(x) \rightarrow g_{R} a_{R}(x) g_{R}^{\dagger}
$$

The field strengths corresponding to local chiral symmetry are

$$
\begin{aligned}
& \hat{F}_{L}^{\mu \nu}=\partial^{\mu} \hat{A}_{L}^{\nu}-\partial^{\nu} \hat{A}_{L}^{\mu}-i\left[\hat{A}_{L}^{\mu}, \hat{A}_{L}^{\nu}\right] \\
& \hat{F}_{R}^{\mu \nu}=\partial^{\mu} \hat{A}_{R}^{\nu}-\partial^{\nu} \hat{A}_{R}^{\mu}-i\left[\hat{A}_{R}^{\mu}, \hat{A}_{R}^{\nu}\right]
\end{aligned}
$$

then, the field strengths of chiral gauge bosons corresponding to global chiral symmetry are

$$
\begin{aligned}
& F_{L}^{\mu \nu}=\partial^{\mu} a_{L}^{\nu}-\partial^{\nu} a_{L}^{\mu}-i\left[a_{L}^{\mu}, a_{L}^{\nu}\right]=\hat{\xi}_{L}(x) \hat{F}_{L}^{\mu \nu} \hat{\xi}_{L}^{\dagger}(x) \\
& F_{R}^{\mu \nu}=\partial^{\mu} a_{R}^{\nu}-\partial^{\nu} a_{R}^{\mu}-i\left[a_{R}^{\mu}, a_{R}^{\nu}\right]=\hat{\xi}_{R}(x) \hat{F}_{R}^{\mu \nu} \hat{\xi}_{R}^{\dagger}(x)
\end{aligned}
$$

All the above field strengths are covariant

$$
\begin{array}{ll}
F_{L}^{\mu \nu} \rightarrow g_{L} F_{L}^{\mu \nu} g_{L}^{\dagger}, & F_{R}^{\mu \nu} \rightarrow g_{R} F_{R}^{\mu \nu} g_{R}^{\dagger} \\
\hat{F}_{L}^{\mu \nu} \rightarrow G_{L} \hat{F}_{L}^{\mu \nu} G_{L}^{\dagger}, & \hat{F}_{R}^{\mu \nu} \rightarrow G_{R} \hat{F}_{R}^{\mu \nu} G_{R}^{\dagger}
\end{array}
$$

Similarly, we can also construct gauge fields as follows

$$
\begin{aligned}
& -\hat{a}_{L}(x) \equiv \xi_{M}(x) i D \xi_{M}^{\dagger}(x)=\xi_{M}(x)\left(i \partial+\hat{A}_{R}(x)\right) \xi_{M}^{\dagger}(x)-\hat{A}_{L}(x) \\
& -\hat{a}_{R}(x) \equiv \xi_{M}^{\dagger}(x) i D \xi_{M}(x)=\xi_{M}^{\dagger}(x)\left(i \partial+\hat{A}_{L}(x)\right) \xi_{M}(x)-\hat{A}_{R}(x)=\xi_{M}^{\dagger}(x) \hat{a}_{L}(x) \xi_{M}((2) .15)
\end{aligned}
$$


they are also covariant under local chiral symmetry

$$
\hat{a}_{L}(x) \rightarrow G_{L}(x) \hat{a}_{L}(x) G_{L}^{\dagger}(x), \quad \hat{a}_{R}(x) \rightarrow G_{R}(x) \hat{a}_{R}(x) G_{R}^{\dagger}(x)
$$

In above, we have defined a set of quantities, but in the sense of gauge fields, there are only two kinds of independent quantities: quantities (hatted quantities) transform according to local chiral symmetry and quantities (unhatted quantities) transform according to global chiral symmetry. They are equivalent in expressing gauge fields since there are only two kinds of gauge fields in our theory. The differences among them are chiral rotated angles. By using these gauge fields and pseudoscalar fields, we can construct chiral, $C, P$ and $T$ invariant lagrangian which consists of pseudoscalar mensons, vector mesons and axial-vector mesons. The Lagrangian, which will be constructed below, should be invariant under the transformations of global chiral symmetry $U(3)_{L} \times U(3)_{R}$ with the local chiral symmetry $\hat{U}(3)_{L} \times \hat{U}(3)_{R}$ appearing as a hidden symmetry.

\section{THE EFFECTIVE LAGRANGIAN OF VECTOR, AXIAL-VECTOR AND PEUSODOSCALAR MESONS}

To construct the Lagrangian, we should take independent quantities from those defined above. By analyzing their transformation properties, the independent quantities may be chosen as follows

$$
a_{L \mu}, \quad U a_{R \mu} U^{\dagger}, \quad \hat{\xi}_{L} \hat{a}_{L \mu} \hat{\xi}_{L}^{\dagger}
$$

They are transforming as $A \rightarrow g_{L} A g_{L}^{\dagger}$ with $A$ denotes the above three quantities.

Thus the $O\left(p^{2}\right)$ Lagrangian can be constructed as

$$
\begin{aligned}
\mathcal{L}^{2} & =\mathcal{L}_{a}^{2}+\mathcal{L}_{b}^{2}+\mathcal{L}_{c}^{2}+\mathcal{L}_{d}^{2} \\
\mathcal{L}_{a}^{2} & =a \operatorname{Tr}\left[a_{L \mu}+U a_{R \mu} U^{\dagger}\right]^{2} \\
\mathcal{L}_{b}^{2} & =b \operatorname{Tr}\left[a_{L \mu}-U a_{R \mu} U^{\dagger}\right]^{2} \\
\mathcal{L}_{c}^{2} & =c \operatorname{Tr}\left[\hat{\xi}_{L} \hat{a}_{L \mu} \hat{a}_{L}^{\mu} \hat{\xi}_{L}^{\dagger}\right] \\
\mathcal{L}_{d}^{2} & =d \operatorname{Tr}\left[\left(a_{L \mu}-U a_{R \mu} U^{\dagger}\right)-\hat{\xi}_{L} \hat{a}_{L \mu} \hat{\xi}_{L}^{\dagger}\right]^{2}
\end{aligned}
$$

where $a, b, c$ and $d$ are constants and will be fixed later. 
When rewriting the lagrangian in the explicit form of chiral angle and covariant derivative and redefining the constants $a, b, c$ and $d$, we have

$$
\begin{aligned}
& \mathcal{L}_{a}^{2}=-a\left(f_{\pi}^{2} / 16\right) \operatorname{Tr}\left[\hat{\xi}_{L} D_{\mu} \hat{\xi}_{L}^{\dagger}+\left(\hat{\xi}_{L} \xi_{M}\right)\left(D_{\mu} \hat{\xi}_{R}^{\dagger}\right) \hat{\xi}_{R}\left(\hat{\xi}_{L} \xi_{M}\right)^{\dagger}\right]^{2} \\
& \mathcal{L}_{b}^{2}=-b\left(f_{\pi}^{2} / 16\right) \operatorname{Tr}\left[\hat{\xi}_{L} D_{\mu} \hat{\xi}_{L}^{\dagger}-\left(\hat{\xi}_{L} \xi_{M}\right)\left(D_{\mu} \hat{\xi}_{R}^{\dagger}\right) \hat{\xi}_{R}\left(\hat{\xi}_{L} \xi_{M}\right)^{\dagger}\right]^{2} \\
& \mathcal{L}_{c}^{2}=-c\left(f_{\pi}^{2} / 16\right) \operatorname{Tr}\left[\xi_{M}^{\dagger} D_{\mu} \xi_{M}\right]^{2} \\
& \mathcal{L}_{d}^{2}=-d\left(f_{\pi}^{2} / 16\right) \operatorname{Tr}\left[\hat{\xi}_{L} D_{\mu} \hat{\xi}_{L}^{\dagger}+\left(\hat{\xi}_{L} \xi_{M}\right)\left(D_{\mu} \hat{\xi}_{R}^{\dagger}\right) \hat{\xi}_{R}\left(\hat{\xi}_{L} \xi_{M}\right)^{\dagger}-\hat{\xi}_{L}\left(\xi_{M} D_{\mu} \xi_{M}^{\dagger}\right) \hat{\xi}_{L}^{\dagger}\right]^{2}
\end{aligned}
$$

which are $O\left(p^{2}\right)$ Lagrangian with $f_{\pi}$ the decay constant.

To construct $O\left(p^{4}\right)$ Lagrangian, let's define some quantities by using the above independent quantities and confirm their parity $(P)$ properties,

$$
\begin{array}{ll}
P: & a_{+\mu} \equiv\left(a_{L \mu}+U a_{R \mu} U^{\dagger}\right) \rightarrow U^{\dagger} a_{+\mu} U \\
P: & a_{-\mu} \equiv\left(a_{L \mu}-U a_{R \mu} U^{\dagger}\right) \rightarrow-U^{\dagger} a_{-\mu} U \\
P: & \hat{a}_{-\mu} \equiv \hat{\xi}_{L} \hat{a}_{L \mu} \hat{\xi}_{L}^{\dagger} \rightarrow-U^{\dagger} \hat{a}_{-\mu} U \\
P: & V_{\mu \nu} \equiv F_{\mu \nu}^{L}+U F_{\mu \nu}^{R} U^{\dagger} \rightarrow U^{\dagger} V_{\mu \nu} U \\
P: & A_{\mu \nu} \equiv F_{\mu \nu}^{L}-U F_{\mu \nu}^{R} U^{\dagger} \rightarrow-U^{\dagger} A_{\mu \nu} U
\end{array}
$$

From the above discussions, the $O\left(p^{4}\right)$ Lagrangian can easily be constructed. A complete Lagrangian is presented in Appendix. Here we focus $\mathcal{L}^{2}$ and the following ten relevant important terms

$$
\begin{aligned}
\mathcal{L}^{4}= & \mathcal{L}_{k}^{4}+\mathcal{L}_{\hat{a}}^{4}+\mathcal{L}_{F}^{4} \\
\mathcal{L}_{k}^{4}= & -\frac{1}{4 g_{G}^{2}} \operatorname{Tr}\left(F_{L \mu \nu} F_{L}^{\mu \nu}+F_{R \mu \nu} F_{R}^{\mu \nu}\right)=-\frac{1}{4 g_{G}^{2}} \operatorname{Tr}\left(\hat{F}_{L \mu \nu} \hat{F}_{L}^{\mu \nu}+\hat{F}_{R \mu \nu} \hat{F}_{R}^{\mu \nu}\right) \\
\mathcal{L}_{\hat{a}}^{4}= & \alpha\left(1 / 12 g_{G}^{2}\right) \operatorname{Tr}\left[\hat{\xi}_{L}\left(D_{\mu} \hat{a}_{L \nu}\right)\left(D^{\mu} \hat{a}_{L}^{\nu}\right) \hat{\xi}_{L}^{\dagger}\right] \\
& +\beta\left(1 / 12 g_{G}^{2}\right) \operatorname{Tr}\left[\hat{\xi}_{L} \hat{a}_{L \mu} \hat{a}_{L \nu} \hat{a}_{L}^{\mu} \hat{a}_{L}^{\nu} \hat{\xi}_{L}^{\dagger}\right] \\
& +\gamma\left(1 / 12 g_{G}^{2}\right) \operatorname{Tr}\left[\left(\hat{\xi}_{L} \hat{a}_{L \mu} \hat{a}_{L}^{\mu} \hat{\xi}_{L}^{\dagger}\right)^{2}\right] \\
\mathcal{L}_{F}^{4}= & \alpha_{1}\left(-i / g_{G}^{2}\right) \operatorname{Tr}\left[a_{L \mu} a_{L \nu} F^{L \mu \nu}+a_{R \mu} a_{R \nu} F^{R \mu \nu}\right] \\
& +\alpha_{2}\left(-i / g_{G}^{2}\right) \operatorname{Tr}\left[U a_{R \mu} a_{R \nu} U^{\dagger} F^{L \mu \nu}+a_{L \mu} a_{L \nu} U F^{R \mu \nu} U^{\dagger}\right] \\
& +\alpha_{3}\left(+i / 2 g_{G}^{2}\right) \operatorname{Tr}\left[a_{L \mu} U a_{R \nu} U^{\dagger} F^{L \mu \nu}+a_{R \mu} U^{\dagger} a_{L \nu} U F^{R \mu \nu}\right]+H . c . \\
& +\alpha_{4}\left(-i / 4 g_{G}^{2}\right) \operatorname{Tr}\left[\hat{\xi}_{L} \hat{a}_{L \mu} \hat{a}_{L \nu} \hat{\xi}_{L}^{\dagger} F^{L \mu \nu}+\hat{\xi}_{R} \xi_{M}^{\dagger} \hat{a}_{L \mu} \hat{a}_{L \nu} \xi_{M} \hat{\xi}_{R}^{\dagger} F^{R \mu \nu}\right]
\end{aligned}
$$




$$
\begin{aligned}
& +\alpha_{5}\left(+i / 4 g_{G}^{2}\right) \operatorname{Tr}\left[a_{L \mu} \hat{\xi}_{L} \hat{a}_{L \nu} \hat{\xi}_{L}^{\dagger} F^{L \mu \nu}+a_{R \mu} \hat{\xi}_{R} \hat{a}_{R \nu} \hat{\xi}_{R}^{\dagger} F^{R \mu \nu}\right]+H . c . \\
& +\alpha_{6}\left(-i / 4 g_{G}^{2}\right) \operatorname{Tr}\left[U a_{R \mu} U^{\dagger} \hat{\xi}_{L} \hat{a}_{L \nu} \hat{\xi}_{L}^{\dagger} F^{L \mu \nu}-U^{\dagger} a_{L \mu} U \hat{\xi}_{R} \hat{a}_{R \nu} \hat{\xi}_{R}^{\dagger} F^{R \mu \nu}\right]+\text { H.c. }
\end{aligned}
$$

For comparison, the coupling constants are taken in terms of the same notations as the ones in ref. 10] 15] except three additional interaction terms of $O\left(p^{4}\right)$ which have been missed in [10] [15] and will be found to be very important for understanding the $\rho \pi \pi$ coupling $g_{\rho \pi \pi}$ and the decay rates of $a_{1} \rightarrow \rho \pi$ and $a_{1} \rightarrow \gamma \pi$. It is seen that there are fourteen unknown coupling constants: $a, b, c, d, g_{G}, \alpha, \beta, \gamma$ and $\alpha_{i}(i=1, \cdots, 6)$. In general, they need to be determined via experimental processes and the success of current algebra can also fix some of the couplings. It was shown in [10] 15] that the following choice of the parameters seem to be consistent with the low energy phenomenology and current algebra

$$
\begin{aligned}
& a=b=c=2, d=0 \\
& \alpha_{1}=\alpha_{2}=\alpha_{3}=0, \quad-\alpha_{4}=\alpha_{5}=\alpha_{6}=1 \\
& \alpha, \beta, \gamma-\text { missed, } \quad \text { or } \quad \alpha=\beta=\gamma=0
\end{aligned}
$$

Note that the values of this set of parameters were phenomenologically suggested including the three terms $\alpha, \beta$ and $\gamma$. The three additional terms are introduced at the first time in this paper from hidden local symmetry. We will discuss their values in the next section in detail.

Since the physics is independent of Hidden Local symmetry, we can choose any appropriate gauges for the local symmetry to obtain the effective Lagrangian for describing the low energy dynamics of QCD. For convenient, we choose the following gauge transformations of $G_{L, R}(x)$ which are the same as [10], so that

$$
\begin{aligned}
& \xi_{M}(x) \rightarrow G_{L}(x) \xi_{M}(x) G_{R}^{\dagger}=1 \\
& \hat{\xi}_{L}(x) \rightarrow \hat{\xi}_{L}(x) G_{L}^{\dagger}(x)=\xi_{L}(x)=\xi(x)=e^{i \Pi(x) / f_{\pi}} \\
& \hat{\xi}_{R}(x) \rightarrow \hat{\xi}_{R}(x) G_{R}^{\dagger}(x)=\xi_{R}(x)=\xi^{\dagger}(x)=e^{-i \Pi(x) / f_{\pi}} \\
& U(x)=\xi_{L}(x) \xi_{R}^{\dagger}(x)=\xi^{2}(x)=e^{i 2 \Pi(x) / f_{\pi}}
\end{aligned}
$$

where $\Pi(x)=\Pi^{a} \lambda^{a}$ is the nonet Goldstone-like pseudoscalar. In our convention, $f_{\pi}=$ 
$186 \mathrm{MeV}$. With this gauge, then we have

$$
\hat{a}_{R}(x)=-\hat{a}_{L}=\hat{A}_{R}-\hat{A}_{L}=\xi_{L}^{\dagger}(-i D U) \xi_{R}=\xi_{R}^{\dagger}\left(i D U^{\dagger}\right) \xi_{L}
$$

where

$$
D U=\partial U+i U a_{R}-i a_{L} U
$$

It is seen that the above choice of gauge condition is a kind of unitary gauge corresponding to the broken down of the hidden local chiral symmetry.

It will also be useful to decompose the chiral gauge fields $\hat{A}_{L}$ and $\hat{A}_{R}$ into two parts

$$
\hat{A}_{L}(x)=A_{L}+L_{\xi}(x), \quad \hat{A}_{R}(x)=A_{R}+R_{\xi}(x)
$$

where $A_{L}(x)$ and $A_{R}(x)$ are the covariant parts associated with the gauge bosons $a_{L}(x)$ and $a_{R}(x)$, while $L_{\xi}(x)$ and $R_{\xi}(x)$ are the pure gauge parts associated with the Goldstone-like pseudoscalars contained in the nonlinear chiral fields $\hat{\xi}_{L}(x)$ and $\hat{\xi}_{R}(x)$

$$
\begin{aligned}
& A_{L}(x)=\xi_{L}^{\dagger}(x) a_{L}(x) \xi_{L}(x) \equiv V(x)-A(x) \\
& L_{\xi}(x)=\xi_{L}^{\dagger}(x) i \partial \xi_{L}(x) \equiv V_{\xi}(x)-A_{\xi}(x) \\
& A_{R}(x)=\xi_{R}^{\dagger}(x) a_{R}(x) \xi_{R}(x) \equiv V(x)+A(x) \\
& R_{\xi}(x)=\xi_{R}^{\dagger}(x) i \partial \xi_{R}(x) \equiv V_{\xi}(x)+A_{\xi}(x)
\end{aligned}
$$

we can explicitly get

$$
2 A_{\xi}=\xi_{L}^{\dagger}(-i \partial U) \xi_{R}
$$

In above gauge, we get the effective Lagrangian which possesses the global $U(3)_{L} \times U(3)_{R}$ symmetry.

The $O\left(p^{2}\right)$ Lagrangian becomes

$$
\begin{aligned}
\mathcal{L}^{2}= & (a+b)\left(f_{\pi}^{2} / 16\right) \operatorname{Tr}\left[a_{L \mu}^{2}+a_{R \mu}^{2}\right]+2(a-b)\left(f_{\pi}^{2} / 16\right) \operatorname{Tr}\left[a_{L \mu} U a_{R \mu} U^{\dagger}\right] \\
& +c\left(f_{\pi}^{2} / 16\right) \operatorname{Tr}\left[D_{\mu} U D^{\mu} U^{\dagger}\right]+d\left(f_{\pi}^{2} / 16\right) \operatorname{Tr}\left[\partial_{\mu} U \partial^{\mu} U^{\dagger}\right]
\end{aligned}
$$

The $O\left(p^{4}\right)$ Lagrangian becomes

$$
\mathcal{L}_{4}=\mathcal{L}_{k}^{4}+\mathcal{L}_{\hat{a}}^{4}+\mathcal{L}_{F}^{4}+\cdots
$$




$$
\begin{aligned}
\mathcal{L}_{k}^{4}= & -\frac{1}{4 g_{G}^{2}} \operatorname{Tr}\left(F_{L \mu \nu} F_{L}^{\mu \nu}+F_{R \mu \nu} F_{R}^{\mu \nu}\right)=-\frac{1}{4 g_{G}^{2}} \operatorname{Tr}\left(\hat{F}_{L \mu \nu} \hat{F}_{L}^{\mu \nu}+\hat{F}_{R \mu \nu} \hat{F}_{R}^{\mu \nu}\right) \\
\mathcal{L}_{\hat{a}}^{4}= & \alpha\left(1 / 12 g_{G}^{2}\right) \operatorname{Tr}\left[D_{\mu} D_{\nu} U D^{\mu} D^{\nu} U^{\dagger}\right] \\
& +\beta\left(1 / 12 g_{G}^{2}\right) \operatorname{Tr}\left[D_{\mu} U D_{\nu} U^{\dagger} D^{\mu} U D^{\nu} U^{\dagger}\right] \\
& +\gamma\left(1 / 12 g_{G}^{2}\right) \operatorname{Tr}\left[D_{\mu} U D^{\mu} U^{\dagger} D_{\nu} U D^{\nu} U^{\dagger}\right] \\
\mathcal{L}_{F}^{4}= & \alpha_{1}\left(-i / g_{G}^{2}\right) \operatorname{Tr}\left[a_{L \mu} a_{L \nu} F^{L \mu \nu}+a_{R \mu} a_{R \nu} F^{R \mu \nu}\right] \\
& +\alpha_{2}\left(-i / g_{G}^{2}\right) \operatorname{Tr}\left[a_{R \mu} a_{R \nu} U^{\dagger} F^{L \mu \nu} U+a_{L \mu} a_{L \nu} U F^{R \mu \nu} U^{\dagger}\right] \\
& +\alpha_{3}\left(+i / 2 g_{G}^{2}\right) \operatorname{Tr}\left[a_{L \mu} U a_{R \nu} U^{\dagger} F^{L \mu \nu}+a_{R \mu} U^{\dagger} a_{L \nu} U F^{R \mu \nu}\right]+H . c . \\
& +\alpha_{4}\left(-i / 4 g_{G}^{2}\right) \operatorname{Tr}\left[D_{\mu} U D_{\nu} U^{\dagger} F^{L \mu \nu}+D_{\mu} U^{\dagger} D_{\nu} U F^{R \mu \nu}\right] \\
& +\alpha_{5}\left(+i / 4 g_{G}^{2}\right) \operatorname{Tr}\left[a_{L \mu} i D_{\nu} U U^{\dagger} F^{L \mu \nu}-a_{R \mu} i D_{\nu} U^{\dagger} U F^{R \mu \nu}\right]+H . c . \\
& +\alpha_{6}\left(-i / 4 g_{G}^{2}\right) \operatorname{Tr}\left[U a_{R \mu} i D_{\nu} U^{\dagger} F^{L \mu \nu}-U^{\dagger} a_{L \mu} i D_{\nu} U F^{R \mu \nu}\right]+H . c .
\end{aligned}
$$

We will see below that it is this form of effective chiral Lagrangian that enables us to compare it with the one derived from effective chiral theory and chiral perturbation theory. This is because they possess the same global chiral symmetry $G_{\text {global }}$ in the chiral limit. It then allows us to fix the fourteen parameters in terms of two parameters introduced in the effective chiral theory of mesons in the large $N_{c}$ approach.

\section{14-PARAMETERS IN CHIRAL LAGRANGIAN OF HIDDEN LOCAL SYM- METRY}

So far, the effective chiral lagrangian based on the global chiral symmetry and local hidden symmetry breaking has been presented. Considering the appropriate gauge selection mentioned in last section, we can fix the 14-parameters by comparing them with the ones of chiral perturbation theory given in [18]. It is easy to check that the parameters are fixed to be

$$
\begin{aligned}
& a=b=\frac{m_{0}^{2}}{f_{\pi}^{2}}=\frac{g^{2} m_{\rho}^{2}}{f_{\pi}^{2}}, \quad c=\frac{6 g^{2} m^{2}}{f_{\pi}^{2}}=\frac{F^{2}}{f_{\pi}^{2}}, \quad d=0 \\
& \alpha=2 \beta=-\gamma=\frac{N_{c}}{2(\pi g)^{2}}, \quad g_{G}^{2}=\frac{4}{g^{2}} \\
& \alpha_{1}=\alpha_{2}=\alpha_{3}=\alpha_{5}=\alpha_{6}=0, \quad \alpha_{4}=\frac{N_{c}}{2(\pi g)^{2}}=\alpha
\end{aligned}
$$


with the redefinition

$$
g^{2}=\frac{1}{6} \frac{F^{2}}{m^{2}}
$$

where $m$ and $m_{0}$ are free parameters.

To define the physical meson states in the mass eigenstates, one needs to normalize the kinetic terms and redefine the pseudoscalars and axial-vectors due to the mixing term $a^{\mu}(x) \partial_{\mu} \pi(x)$, which leads to

$$
\begin{aligned}
& f_{\pi}^{2}=F^{2}\left(1-\frac{2 c}{a+b+2 c}\right)=F^{2}\left(1-\frac{6 m^{2}}{m_{\rho}^{2}+6 m^{2}}\right) \\
& m_{\rho}^{2}=m_{o}^{2} / g^{2}
\end{aligned}
$$

Comparing the above parameters with the one fixed via the low energy phenomenology, we can get the following conclusions:

(1). For the terms in the effective Lagrangian up to the $O\left(p^{2}\right)$, both effective chiral Lagrangian approach and hidden local symmetry approach provide a consistent determination for the four parameters. It is interesting to note that once the vector mass is dynamically generated and takes the value

$$
m_{\rho}^{2}=6 m^{2}
$$

we have from (4.1) and (4.3)

$$
F^{2}=2 f_{\pi}^{2}, \quad a=b=c=2
$$

which agree well with the conclusions obtained from the current algebra and phenomenology analysis in the hidden symmetry approach [10] 15].

(2). From the terms in $O\left(p^{4}\right)$, we noticed that: (i) there are ten important terms rather than seven terms in the usual effective Lagrangian of hidden symmetry in [10] [15], three additional new terms (i.e., $\alpha, \beta$ and $\gamma$ ) are necessary in our present more general construction on effective Lagrangian via the hidden local symmetry approach. Of particular, these three terms are found to nonzero when comparing with the effective chiral Lagrangian [18]; (ii) Even for the usual six terms with coupling constants $\alpha_{i}, i=1, \cdots, 6$, three of the them, $\alpha_{4}, \alpha_{5}$ and $\alpha_{6}$, turn out to have different behavior when comparing their values yielded from the phenomenological analysis in the literature [10] [15] with the ones determined from the effective chiral theory[18]. 
(3). The relation $-\alpha_{4}=\alpha_{5}=\alpha_{6}=1$ has been taken in the literature [10] 15] to accommodate the $\rho$-dominance for $a_{1} \rightarrow \gamma \pi$ decay and cancel the strong momentum dependence of the coupling $f_{\rho \pi \pi}$ in the absence of $a_{1}$-meson. While in the effective chiral theory, it is seen that $\alpha_{4}$ is positive with the value $\alpha_{4}=N_{c} /\left(2(\pi g)^{2}\right)$ and $\alpha_{5}=\alpha_{6}=0$.

(4). It is natural to ask why the values $\alpha_{4}, \alpha_{5}$ and $\alpha_{6}$ extracted from the two cases are so different, and how the cancellation of strong momentum dependence of the coupling $f_{\rho \pi \pi}$ and $\rho$-dominance in $a_{1} \rightarrow \gamma \pi$ decay can be accommodated in the case with positive value of $\alpha_{4}$ and zero values of $\alpha_{5}$ and $\alpha_{6}$. The answer is attributed to three additional new terms in our present more general construction from hidden local symmetry approach. They are found to be nonzero from the effective chiral theory and their values are determined from the effective chiral theory to be $\alpha=-\gamma=2 \beta=\alpha_{4}=N_{c} /\left(2(\pi g)^{2}\right)$. With these values, it can be shown that the strong momentum dependence of $f_{\rho \pi \pi}$ will be cancelled when $m_{\rho}^{2}=6 \mathrm{~m}^{2}$ and $g=1 / \pi$ due to the existence of additional new terms, and the $\rho$-dominance for $a_{1} \rightarrow \gamma \pi$ decay can also be realized [18].

(5). In comparison with the chiral perturbation theory (ChPT) 2], the new terms $\alpha, \beta$ and $\gamma$ are related to the terms $L_{1}, L_{2}$ and $L_{3}$ in ChPT. Noticing the algebraic relation

$$
\begin{aligned}
& \operatorname{Tr}\left(D_{\mu} U D_{\nu} U^{\dagger} D^{\mu} U D^{\nu} U^{\dagger}\right)=\frac{1}{2}\left[\operatorname{Tr}\left(D_{\mu} U D^{\mu} U^{\dagger}\right)\right]^{2} \\
& +\operatorname{Tr}\left(D_{\mu} U D_{\nu} U^{\dagger}\right) \cdot \operatorname{Tr}\left(D^{\mu} U D^{\nu} U^{\dagger}\right)-2 \operatorname{Tr}\left(D_{\mu} U D^{\mu} U^{\dagger}\right)^{2}
\end{aligned}
$$

we get the relation $L_{1}=1 / 2 L_{2}$. According to (4.6) and the following (5.7), we can express $L_{1}, L_{2}$ and $L_{3}$ in terms of $\alpha, \beta$ and $\gamma$ as:

$$
L_{1}=\frac{\beta}{2} \frac{1}{12 g_{G}^{2}}, \quad L_{2}=\beta \frac{1}{12 g_{G}^{2}}, \quad L_{3}=(\alpha-2 \beta+\gamma) \frac{1}{12 g_{G}^{2}}, \quad L_{9}=\left(3 \alpha_{4}-\alpha\right) \frac{1}{12 g_{G}^{2}}
$$

(6). The terms $\alpha_{4}$ and $\alpha$ are related to the coupling constants $L_{9}$ in ChPT. Both the sign and extracted value for $\alpha_{4}$ in our present considerations are consistent with the ones of $L_{9}$ from the phenomenology well described by ChPT, while the previous results for $\alpha_{4}$ given in literature 10] 15] seem to be conflict with the extracted value of $L_{9}$ in ChPT.

It is then not difficult to show that the more general effective Lagrangian constructed via the approach of global chiral symmetry and hidden local chiral symmetry with an appropriate gauge choice should be consistent with any other effective chiral Lagrangian in the chiral limit. The fourteen parameters in the effective Lagrangian up to $O\left(p^{4}\right)$ of the mesons fields can be extracted from the effective chiral theory. 


\section{EFFECTIVE CHIRAL LAGRANGIAN AND LOW ENERGY BEHAVIOR}

A consistent effective Lagrangian should reproduce the low energy phenomenologies which have been tested by experiments. Now, let's check the Vector-Pseudoscalar-Pseudoscalar vertex. As an example, we may first work out the $\rho \pi \pi$ coupling $f_{\rho \pi \pi}$ which is defined as

$$
\mathcal{L}_{\rho \pi \pi}=f_{\rho \pi \pi} \epsilon_{i j k} \rho_{i}^{\mu} \pi_{j} \partial_{\mu} \pi_{k}
$$

From the general Lagrangian (3.26) and (3.28), it is easy to get

$$
\begin{aligned}
f_{\rho \pi \pi}= & g_{G}\left\{1+\frac{2 m_{\rho}^{2}}{g_{G}^{2} f_{\pi}^{2}}\left[\left(\alpha_{4}-\alpha / 3\right)\left(1-\frac{2 c}{a+b+2 c}\right)^{2}-\left(\frac{2 c}{a+b+2 c}\right)^{2}\right.\right. \\
& \left.\left.+\left(\alpha_{5}+\alpha_{6}\right)\left(\frac{2 c}{a+b+2 c}\right)\left(1-\frac{2 c}{a+b+2 c}\right)\right]\right\}
\end{aligned}
$$

from the above expression we can see that there is contribution from $\alpha$. It is seen that when the parameters take the values chosen from the phenomenological analysis in [10] [15], i.e., $a=b=c=2,-\alpha_{4}=\alpha_{5}=\alpha_{6}=1$ and $\alpha=0$, one has

$$
f_{\rho \pi \pi}=g_{G}=2 / g
$$

where the second term in the curled bracket of (5.2) vanishes due to cancellations from various contributions. Alternatively, we can take other choices, such as the values in [18]. As a consequence, we have

$$
f_{\rho \pi \pi}=\frac{2}{g}\left\{1+\frac{m_{\rho}^{2}}{2 \pi^{2} f_{\pi}^{2}}\left[\frac{N_{c}}{3}\left(1-\frac{6 m^{2}}{m_{\rho}^{2}+6 m^{2}}\right)^{2}-\pi^{2} g^{2}\left(\frac{6 m^{2}}{m_{\rho}^{2}+6 m^{2}}\right)^{2}\right]\right\}
$$

It is seen that only for a specific choice $g=1 / \pi, m_{\rho}^{2}=6 m^{2}$ and $N_{c}=3$, we get $f_{\rho \pi \pi}=2 / g$. It can be shown that with the parameters fixed from the effective chiral theory, the effective chiral Lagrangian can also lead to a consistent prediction on $\Gamma\left(a_{1} \rightarrow \rho \pi\right)$ and $\Gamma\left(a_{1} \rightarrow \gamma \pi\right)$. The numerical result were found to be $\Gamma\left(a_{1} \rightarrow \rho \pi\right) \simeq 326 \mathrm{MeV}$ and $\Gamma\left(a_{1} \rightarrow \gamma \pi\right) \simeq 252 \mathrm{KeV}$. In general, the value of the basic parameter $g$ closing to $1 / \pi$ is found to be a consistent one. Here the term $\alpha$ plays an important role.

The second effect of the additional term $\alpha$ in the more general effective chiral Lagrangian is that the weinberg's sum rule $g_{a}^{2}=g_{\rho}^{2}$ will be modified to be

$$
g_{a}^{2}=g_{\rho}^{2}\left(1-\frac{\alpha}{3}\right)=g_{\rho}^{2}\left(1-\frac{N_{c}}{6 \pi^{2} g^{2}}\right)
$$


where $g_{a}$ and $g_{\rho}$ were defined in [18]. In the second equation, the parameter $\alpha$ has been taken the result fixed from the effective chiral theory. This modification makes the predictions for the masses of the axial-vectors to be more consistent with the experimental data.

The third important effect from the additional term $\alpha$ is the evaluation for the decay constants of the pseudoscalars.

To be more explicit, we may use some algebraic relations and equation of motion

$$
D^{\mu}\left(U^{\dagger} D_{\mu} U\right)=\frac{1}{2}\left(U^{\dagger} \chi-\chi^{\dagger} U\right)-\frac{1}{6} \operatorname{Tr}\left(U^{\dagger} \chi-\chi^{\dagger} U\right)
$$

to reexpress the $\alpha$ term into several more familiar terms, so that its effect can be easily seen. It is easy to check that

$$
\begin{aligned}
D_{\mu} D_{\nu} U D^{\mu} D^{\nu} U^{\dagger}= & \frac{1}{2}\left[F_{L}^{2}+F_{R}^{2}-2 F_{L} U F_{R} U^{\dagger}\right] \\
& +i\left[D_{\mu} U D_{\nu} U^{\dagger} F_{L}^{\mu \nu}+D_{\mu} U^{\dagger} D_{\nu} U F_{R}^{\mu \nu}\right] \\
& +\left(D_{\mu} U D^{\mu} U^{\dagger}\right)\left(D_{\nu} U D^{\nu} U^{\dagger}\right) \\
& +\frac{1}{2} D_{\mu} U D^{\mu}\left[U^{\dagger}\left(U \chi^{\dagger}-\chi^{\dagger} U^{\dagger}\right)\right] \\
& \left.+\frac{1}{2}\left(D_{\mu} U^{\dagger} D^{\mu} U\right)\left[U^{\dagger} \chi-\chi^{\dagger} U\right)\right] \\
& + \text { total derivative terms or trace terms }
\end{aligned}
$$

From the explicit form, it is not difficult to understand its effects. Where the first term modifies the Weinberg's sum rule, the second term contributes to the $\rho \pi \pi$ coupling and the coupling constant $L_{9}$ in ChPT, the third term has effects on the coupling constant $L_{3}$ in ChPT and the last two terms will provide additional contributions to the decay constants of psuedoscalars. As a consequence, we arrive at a complete prediction for the coupling $L_{1}, L_{2}, L_{3}$ and $L_{9}$ at this order, which is consistent with the ones extracted from phenomenology described by the chiral perturbation theory up to $O\left(p^{4}\right)$. The numerical values are found to be

\begin{tabular}{|c|c|c|c|c|}
\hline Parameters & $10^{3} L_{1}$ & $10^{3} L_{2}$ & $10^{3} L_{3}$ & $10^{3} L_{9}$ \\
\hline Present & 0.79 & 1.58 & -3.16 & 6.32 \\
\hline ChPT[5] & $0.4 \pm 0.3$ & $1.35 \pm 0.3$ & $-3.5 \pm 1.1$ & $6.9 \pm 0.7$ \\
\hline
\end{tabular}

Now, let's check the known KSFR relation. From the general effective Lagrangian, the mass of $\rho$ meson is expressed as $m_{\rho}^{2}=a f_{\pi}^{2} g_{G}^{2} / 4$. Comparing with the effective chiral theory 
with $g_{G}^{2}=4 / g^{2}$ and $f_{\rho \pi \pi} \simeq 2 / g$, one has

$$
m_{\rho}^{2}=a f_{\pi}^{2} g_{G}^{2} / 4=\frac{a}{4} f_{\pi}^{2}\left(\frac{2}{g}\right)^{2} \simeq \frac{a}{4} f_{\pi}^{2} f_{\rho \pi \pi}^{2}
$$

Thus the known KSFR relation holds for $a \simeq 2$ which is also consistent with vector meson dominance.

It is seen that the more general effective Lagrangian with its parameters extracted from the effective chiral theory can well reproduce the phenomenologies of $\rho-\pi$ system.

The fourth effect of the new terms is the important contributions to the $\pi-\pi$ scattering 2] 17] [18].

One may see that only from the $\rho \pi \pi$ coupling, $a_{1} \rightarrow \rho \pi$ and $a_{1} \rightarrow \gamma \pi$ decays, the parameters appearing in the $O\left(p^{4}\right)$ in the effective chiral Lagrangian constructed from the hidden local symmetry approach may not uniquely be determined. The value of parameter $\alpha_{4}$ extracted from the phenomenology of $\rho-a_{1}$ system in the literatures [10] 15] is conflict with the one from the phenomenology well described by the chiral perturbation theory and effective chiral theory. While the resulting structure and couplings from the effective chiral theory are consistent not only with the phenomenology of $\rho-a_{1}$ system, but also with the chiral perturbation theory. Thus, the effective chiral theory derived from the chiral quarks and bound state solutions of nonperturbative QCD may provide a very useful way to extract all the parameters in terms of only two basic scales $m$ and $f_{\pi}=186 \mathrm{MeV}$ (or

coupling constant g). It is likely that the structure of the effective chiral Lagrangians for the $O\left(p^{4}\right)$ given in literatures [10] 15] is incomplete. As a consequence, the extracted coupling constant are not reliable.

\section{CONCLUSIONS}

The more general effective chiral Lagrangian of mesons (pseudoscalars, vectors and axialvectors) has been constructed in the chiral limit by using explicit global chiral symmetry $U(3)_{L} \times U(3)_{R}$ and hidden local chiral symmetry $\hat{U}(3)_{L} \times \hat{U}(3)_{R}$. It is shown that there are many extra terms in addition to the eleven terms given in paper [10]. Among these extra terms there are three important terms that have been found to play important roles in understanding the vector meson dominance and the $\pi-\pi$ scattering, in providing consistent predictions on the decay rates of $a_{1} \rightarrow \rho \pi$ and $a_{1} \rightarrow \gamma \pi$, as well as in resulting a consistent 
effective chiral Lagrangian with the chiral perturbation theory.

It is observed that not only the three new interactional terms introduced in this paper are necessary, but also the resulting coupling constants for other three interacting terms have total different values in comparison with the ones given in the literature from hidden symmetry approach to $O\left(p^{2}\right)$. It is likely that the structure of the effective Lagrangian to $O\left(p^{4}\right)$ given in literature [10] is incomplete, thus the extracted coupling constants are not reliable.

\section{Acknowledgments}

We would like to thank B.A.Li for his valuable discussions. This work was supported in part by the key projects of Chinese Academy of Sciences, the National Science Foundation of China (NSFC).

\section{APPENDIX A: THE FULL LAGRANGIAN TO $O\left(p^{4}\right)$}

In general, the $O\left(p^{4}\right)$ lagrangian has two forms which corresponding to one trace operator terms and two trace operator terms. One trace operator terms are constructed by basic blocks while two trace operator terms are constructed by two $O\left(p^{2}\right)$ terms. As the two trace terms corresponding higher order contributions, we will not consider those terms. For convenience, we construct the $O\left(p^{4}\right)$ Lagrangian from the parity properties of the independent fields.

(i), terms independent of $\hat{a}_{-},\left(\hat{a}_{-\mu} \equiv \hat{\xi}_{L} \hat{a}_{L \mu} \hat{\xi}_{L}^{\dagger}\right)$

$$
\begin{aligned}
\mathcal{L}_{a}^{4}= & a_{1} \operatorname{Tr}\left[\left(a_{\mu-} a_{-}^{\mu}\right)^{2}\right]+a_{2} \operatorname{Tr}\left[a_{\mu-} a_{\nu-} a_{-}^{\mu} a_{-}^{\nu}\right]+a_{3} \operatorname{Tr}\left[\left(a_{\mu+} a_{+}^{\mu}\right)^{2}\right]+a_{4} \operatorname{Tr}\left[a_{\mu+} a_{\nu+} a_{+}^{\mu} a_{+}^{\nu}\right] \\
& +a_{5} \operatorname{Tr}\left[a_{\mu-} a_{-}^{\mu} a_{\nu+} a_{+}^{\nu}\right]+a_{6} \operatorname{Tr}\left[a_{\mu-} a_{\nu-} a_{+}^{\mu} a_{+}^{\nu}\right]+a_{7} \operatorname{Tr}\left[a_{\mu-} a_{\nu-} a_{+}^{\nu} a_{+}^{\mu}\right] \\
& +a_{8}\left\{\operatorname{Tr}\left[a_{\mu-} a_{+}^{\mu} a_{\nu-} a_{+}^{\nu}\right]+\operatorname{Tr}\left[a_{\mu+} a_{-}^{\mu} a_{\nu+} a_{-}^{\nu}\right]\right\}+a_{9} \operatorname{Tr}\left[a_{\mu-} a_{\nu+} a_{-}^{\mu} a_{+}^{\nu}\right]
\end{aligned}
$$

(ii), terms depend on $\hat{a}_{-}$

$$
\begin{aligned}
\mathcal{L}_{\hat{a}}^{4}= & \hat{a}_{1} \operatorname{Tr}\left[a_{\mu-} a_{\nu-} a_{-}^{\mu} \hat{a}_{-}^{\nu}\right]+\hat{a}_{2} \operatorname{Tr}\left[a_{\mu-} a_{-}^{\mu} a_{\nu-} \hat{a}_{-}^{\nu}\right]+\hat{a}_{3} \operatorname{Tr}\left[a_{\nu-} a_{\mu-} a_{-}^{\mu} \hat{a}_{-}^{\nu}\right] \\
& +\hat{a}_{4} \operatorname{Tr}\left[a_{\mu-} a_{\nu-} \hat{a}_{-}^{\mu} \hat{a}_{-}^{\nu}\right]+\hat{a}_{5} \operatorname{Tr}\left[a_{\mu-} a_{-}^{\mu} \hat{a}_{-\nu} \hat{a}_{-}^{\nu}\right]+\hat{a}_{6} \operatorname{Tr}\left[a_{\mu-} a_{\nu-} \hat{a}_{-}^{\nu} \hat{a}_{-}^{\mu}\right]
\end{aligned}
$$




$$
\begin{aligned}
& +\hat{a}_{7} \operatorname{Tr}\left[a_{\mu-} \hat{a}_{-}^{\mu} a_{\nu-} \hat{a}_{-}^{\nu}\right]+\hat{a}_{8} \operatorname{Tr}\left[a_{\mu-} \hat{a}_{-\nu} a_{-}^{\mu} \hat{a}_{-}^{\nu}\right]+\hat{a}_{9} \operatorname{Tr}\left[a_{\mu-} \hat{a}_{-}^{\nu} a_{\nu-} \hat{a}_{-}^{\mu}\right] \\
& +\hat{a}_{10} \operatorname{Tr}\left[a_{\mu-} \hat{a}_{-\nu} \hat{a}_{-}^{\mu} \hat{a}_{-}^{\nu}\right]+\hat{a}_{11} \operatorname{Tr}\left[a_{\mu-} \hat{a}_{-}^{\mu} \hat{a}_{-\nu} \hat{a}_{-}^{\nu}\right]+\hat{a}_{12} \operatorname{Tr}\left[a_{\mu-} \hat{a}_{-\nu} \hat{a}_{-}^{\nu} \hat{a}_{-}^{\mu}\right] \\
& +\hat{a}_{13} \operatorname{Tr}\left[a_{\mu+} a_{\nu+} \hat{a}_{-}^{\mu} \hat{a}_{-}^{\nu}\right]+\hat{a}_{14} \operatorname{Tr}\left[a_{\mu+} a_{+}^{\mu} \hat{a}_{-\nu} \hat{a}_{-}^{\nu}\right]+\hat{a}_{15} \operatorname{Tr}\left[a_{\mu+} a_{\nu+} \hat{a}_{-}^{\nu} \hat{a}_{-}^{\mu}\right] \\
& +\hat{a}_{16} \operatorname{Tr}\left[a_{\mu+} \hat{a}_{-\nu} a_{+}^{\mu} \hat{a}_{-}^{\nu}\right]+\hat{a}_{17} \operatorname{Tr}\left[a_{\mu+} \hat{a}_{-}^{\mu} a_{\nu+} \hat{a}_{-}^{\nu}\right]+\hat{a}_{18} \operatorname{Tr}\left[a_{\mu+} \hat{a}_{-\nu} a_{+}^{\nu} h^{\mu}\right] \\
& +\hat{a}_{19} \operatorname{Tr}\left[\hat{a}_{-\mu} a_{-}^{\mu} a_{\nu+} a_{+}^{\nu}\right]+\hat{a}_{20} \operatorname{Tr}\left[\hat{a}_{-\mu} a_{\nu-} a_{+}^{\mu} a_{+}^{\nu}\right]+\hat{a}_{21} \operatorname{Tr}\left[\hat{a}_{-\mu} a_{-}^{\nu} a_{\nu+} a_{+}^{\mu}\right] \\
& +\hat{a}_{22} \operatorname{Tr}\left[a_{\mu-} h^{\mu} a_{\nu+} a_{+}^{\nu}\right]+\hat{a}_{23} \operatorname{Tr}\left[a_{\mu-} \hat{a}_{-\nu} a_{+}^{\nu} a_{+}^{\mu}\right]+\hat{a}_{24} \operatorname{Tr}\left[a_{\mu-} \hat{a}_{-\nu} a_{+}^{\mu} a_{+}^{\nu}\right] \\
& +\hat{a}_{25} \operatorname{Tr}\left[a_{\mu-} a_{\nu+} \hat{a}_{-}^{\mu} a_{+}^{\nu}\right]+\hat{a}_{26} \operatorname{Tr}\left[a_{\mu-} a_{+}^{\nu} \hat{a}_{-\nu} a_{+}^{\mu}\right]+\hat{a}_{27} \operatorname{Tr}\left[a_{\mu-} a_{+}^{\mu} \hat{a}_{-\nu} a_{+}^{\nu}\right] \\
& +\hat{a}_{28} \operatorname{Tr}\left[\hat{a}_{-\mu} \hat{a}_{-\nu} \hat{a}_{-}^{\mu} \hat{a}_{-}^{\nu}\right]+\hat{a}_{29} \operatorname{Tr}\left[\left(\hat{a}_{-\mu} \hat{a}_{-}^{\mu}\right)^{2}\right]
\end{aligned}
$$

(iii), terms depend on $V_{\mu \nu}$ and $A_{\mu \nu}$

$$
\begin{aligned}
\mathcal{L}_{F}^{4}= & \alpha_{1}\left(-i / g_{G}^{2}\right) \operatorname{Tr}\left[a_{L \mu} a_{L \nu} F^{L \mu \nu}+a_{R \mu} a_{R \nu} F^{R \mu \nu}\right] \\
& +\alpha_{2}\left(-i / g_{G}^{2}\right) \operatorname{Tr}\left[U a_{R \mu} a_{R \nu} U^{\dagger} F^{L \mu \nu}+a_{L \mu} a_{L \nu} U F^{R \mu \nu} U^{\dagger}\right] \\
& +\alpha_{3}\left(i / 2 g_{G}^{2}\right) \operatorname{Tr}\left[a_{L \mu} U a_{R \nu} U^{\dagger} F^{L \mu \nu}+a_{R \mu} U^{\dagger} a_{L \nu} U F^{R \mu \nu}\right]+H . c . \\
& +\alpha_{4}\left(-i / 4 g_{G}^{2}\right) \operatorname{Tr}\left[\hat{\xi}_{L} \hat{a}_{L \mu} \hat{a}_{L \nu} \hat{\xi}_{L}^{\dagger} F^{L \mu \nu}+\hat{\xi}_{R} \xi_{M}^{\dagger} \hat{a}_{L \mu} \hat{a}_{L \nu} \hat{\xi}_{M} \hat{\xi}_{R}^{\dagger} F^{R \mu \nu}\right] \\
& +\alpha_{5}\left(+i / 4 g_{G}^{2}\right) \operatorname{Tr}\left[a_{L \mu} \hat{\xi}_{L} \hat{a}_{L \nu} \hat{\xi}_{L}^{\dagger} F^{L \mu \nu}+a_{R \mu} \hat{\xi}_{R} \hat{a}_{R \nu} \hat{\xi}_{R}^{\dagger} F^{R \mu \nu}\right]+H . c . \\
& +\alpha_{6}\left(-i / 4 g_{G}^{2}\right) \operatorname{Tr}\left[U a_{R \mu} U^{\dagger} \hat{\xi}_{L} \hat{a}_{L \nu} \hat{\xi}_{L}^{\dagger} F^{L \mu \nu}+U^{\dagger} a_{L \mu} U \hat{\xi}_{R} \hat{a}_{R \nu} \hat{\xi}_{R}^{\dagger} F^{R \mu \nu}\right]+H . c .
\end{aligned}
$$

After taking the unitary gauge used in the context, the final Lagrangian can be easily written down, we shall not list the full results here.

[1] S.Weinberg, Physica A96, 327 (1979).

[2] J.Gasser and H.Leutwyler, Annals phys. 158, 142 (1984).

J.Gasser and H.Leutwyler, Nucl. Phys. B250, 465 (1985).

[3] J.Wess and B.Zumino, Phys. Lett.B 37, 95(1971).

[4] E.Witten, Nucl. Phys. B 223, 422(1983).

[5] G.Ecker, J.Gasser, H.Leutwyler, A.Pich and E. de Rafael, Phys. Lett. B 223425 (1989).

[6] O.Kaamakcalam, S.Rajeev, J.Schechter, Phys. Rev. D 30, 594(1984).

[7] U.G.Meissner, Phys. Rept.161 213(1988), and references therein. 
[8] G.Ecker, J.Gasser, A.Pich and E. de Rafael, Nucl. Phys. B 321311 (1989).

[9] M. Bando, T.Kugo, S.Uehara, K.Yamawaki, T.Yanagida, Phys. Rev. Lett. 54, 1215 (1985).

[10] M.Bando, T.Kugo and K.Yamawaki, Phys.Rept.164, 217 (1988), and references therein.

[11] M.Harada and K.Yamawaki Phys.Rept.381,1-233(2003), hep-ph/0302103.

[12] Xue-Lei Wang and Qing Wang, Commun.Theor.Phys.34, 519-530(2000).

[13] Y.B. Dai and Y.L. Wu, Eur.Phys. J C (2004) (DOI) 10.1140/epjcd/s2004-01-001-3, hep-ph/0304075.

[14] Y.L. Wu, Int. J. Mod. Phys. A18 , 5363 (2003).

[15] M.Bando, T.Fujiwara and K.Yamawaki, Prog. Theor. Phys. 791140 (1988).

[16] J.F.Donoghue, C.Ramirez and G.Valencia, Phys. Rev. D 39, 1947(1989).

[17] J.Bijnens, G.Colangelo, G.Ecker, J.Gasser, M.E.Sainio, Nucl.Phys.B 508, 263 (1997), Erratum-ibid.B 517,639 (1998).

J.Bijnens, G.Colangelo, G.Ecker, J.Gasser, M.E.Sainio, Phys.Lett.B 374, 210 (1996)

[18] B.A.Li, Phys. Rev. D52 5165 (1995); D52 5184 (1995), and reference therein. 